in $B$ cells $(p=3 e-26), C D 8^{+} T$ cells $(3 e-12)$ and $C D 4^{+} T$ cells $(\mathrm{p}=9 \mathrm{e}-10)$.

A trans-pQTL for IFNGR1 level in monocytes (rs1801274 in FCGR2A; $\mathrm{p}=3 \mathrm{e}-23$ ) and a suggestive significant $\mathrm{pQTL}$ $(\mathrm{p}<5.0 \mathrm{e}-5)$ for IFN- $\gamma$-induced STAT1 phosphorylation in monocytes (rs912784 in LRRC63) were previously associated with SLE. Notably, none of the SNPs in TYK2, STAT4 or IRF5 reached the suggestive significant levels for the parameters studied, and no enrichment of SLE-associated SNPs were identified among pQTLs.

Conclusions We demonstrate a cell-type and stimuli-specific genetic regulation of the IFN system. Two SNPs previously linked to SLE were associated with alterations in the IFN- $\gamma$ receptor expression or response. Further studies to determine the underlying mechanisms of these associations are ongoing.

\section{O24 FLARE ASSESSMENTS IN PATIENTS WITH ACTIVE SYSTEMIC LUPUS ERYTHEMATOSUS (SLE) TREATED WITH ANIFROLUMAB IN 2 PHASE 3 TRIALS}

${ }^{1}$ Richard Furie, ${ }^{2}$ Eric $\mathrm{F}$ Morand, ${ }^{3}$ Anca Askanase, ${ }^{4,5} \mathrm{Ed}$ Vital, ${ }^{6}$ Rubana N Kalyani, ${ }^{7}$ Gabriel Abreu, ${ }^{6}$ Lilia Pineda, ${ }^{6}$ Raj Tummala. 'Zucker School of Medicine at Hofstral Northwell, Great Neck, NY, USA; ${ }^{2}$ Monash University, Melbourne, VIC, Australia; ${ }^{3}$ Columbia University College of Physicians and Surgeons, New York, NY, USA; ${ }^{4}$ University of Leeds, Leeds, UK; ${ }^{5}$ NIHR Leeds Biomedical Research Centre at Leeds Teaching Hospitals NHS Trust, Leeds, UK; ${ }^{6}$ AstraZeneca, Gaithersburg, MD, USA; ${ }^{7}$ AstraZeneca, Gothenburg, Sweden

10.1136/lupus-2020-eurolupus.35

Background Anifrolumab treatment improved BICLA response rates in patients with SLE in the phase 3 TULIP-2 and
TULIP-1 trials (Morand et al, 2020; Furie et al, 2019). In addition, annualized flare rates were lower with anifrolumab vs placebo. TULIP-2 and TULIP-1 data were analyzed to assess effects of anifrolumab on the number of flares and time to first flare during 52 weeks of treatment.

Methods The randomized, double-blind, placebo-controlled TULIP-2 and TULIP-1 trials evaluated efficacy and safety of intravenous anifrolumab (300 mg Q4W) over 52 weeks in patients with moderate to severe SLE despite standardof-care treatment. Flares were defined as $\geq 1$ new BILAG2004 A or $\geq 2$ new (worsening) BILAG-2004 B domain scores compared with the prior month's visit. Number of flares, time to first flare, and annualized flare rate were assessed.

Results In TULIP-2 (anifrolumab, $\mathrm{n}=180$; placebo, $\mathrm{n}=182$ ) and TULIP-1 (anifrolumab, $\mathrm{n}=180$; placebo, $\mathrm{n}=184$ ), fewer anifrolumab-treated patients experienced $\geq 1$ flare (TULIP-2: 31.1\%, $\mathrm{n}=56$; TULIP-1: $36.1 \%, \mathrm{n}=65)$ vs placebo-treated patients (TULIP-2: 42.3\%, $\mathrm{n}=77$; TULIP-1: $43.5 \%, \mathrm{n}=80$ ). Results favoring anifrolumab were observed in time to first flare (TULIP-2: hazard ratio $[\mathrm{HR}]=0.65,95 \%$ confidence interval [CI] 0.46-0.91; TULIP-1: HR $=0.76$, 95\% CI $0.55-$ 1.06; figure 1) and annualized flare rates (TULIP-2: adjusted rate ratio $=0.67,95 \%$ CI $0.48-0.94 ;$ TULIP-1: rate ratio $=0.83,95 \%$ CI $0.60-1.14)$.

Conclusions Across 2 phase 3 trials, we observed reduced total number of flares and annualized flare rates, as well as prolongation of time to first flare with anifrolumab treatment vs placebo. These results support the potential of anifrolumab to reduce disease activity and reduce flares, benefiting patients with SLE.

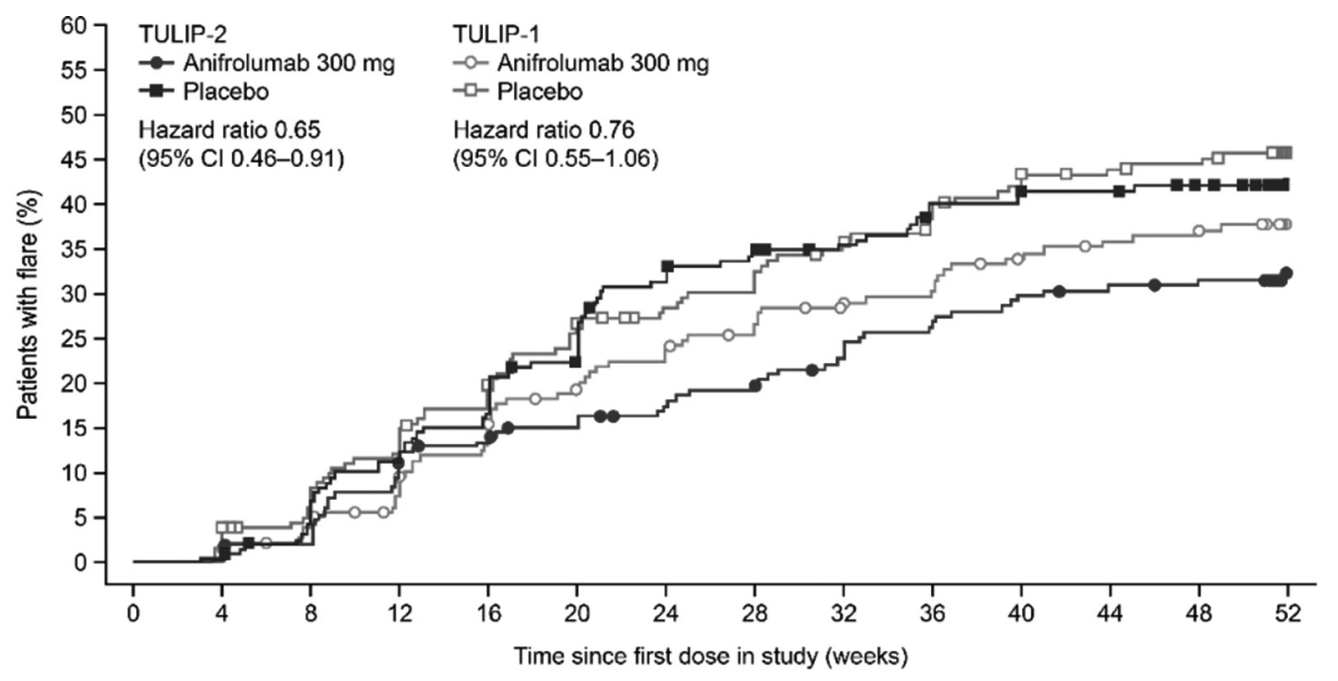

\begin{tabular}{|rllllllllllllll|}
\hline \begin{tabular}{|} 
Number at risk in TULIP-2 \\
Anifrolumab 300 mg
\end{tabular} 180 & 179 & 175 & 158 & 153 & 147 & 141 & 138 & 130 & 124 & 118 & 116 & 114 & 103 \\
Placebo & 182 & 181 & 167 & 159 & 149 & 137 & 120 & 113 & 107 & 98 & 97 & 95 & 91 & 77 \\
\hline
\end{tabular}

BILAG, British Isles Lupus Assessment Group.

Note: Flare defined as $\geq 1$ new BILAG-2004 A or $\geq 2$ new (worsening) BILAG-2004 B domain scores as compared with the previous visit. Time to first flare is derived as date of first flare minus date of first administration of investigational product. If the patient did not have a flare, the time to flare is censored at the end of the exposure time. Time to first flare was evaluated using a Cox proportional-hazards model. 